Eltern-Kind-Kontakt

\section{Hautkontakt nach der Geburt schützt vor Klinikaufnahmen}

Agudelo $S$ et al. Effect of skin-to-skin contact at birth on early neonatal hospitalization. Early Hum Dev 2020; 144: 105020. doi:10.1016/j. earlhumdev.2020.105020

Nach der Geburt stabile Neugeborene sollen gemäß einer Empfehlung der Weltgesundheitsorganisation mindestens eine Stunde lang auf der Brust ihre Mutter liegen, da dies die Mutter-Kind-Bindung und die Stillbeziehung fördert, kardiorespiratorisch und thermostabilisierend wirkt sowie die Kortisolspiegel günstig beeinflusst. Schützt der Hautkontakt nach der Geburt auch vor neonatalen Komplikationen und Hospitalisationen?

Diese Fragestellung untersuchte ein Forscherteam aus Kolumbien mithilfe einer retrospektiven Kohortenstudie. Die Wissenschaftlerinnen und Wissenschaftler werteten die Daten von 816 Neugeborenen aus, welche per Vaginalgeburt mit einem Geburtsgewicht von mindestens $2000 \mathrm{~g}$ zur Welt gekommen waren und eine problemlose kardiorespiratorische Anpassung gezeigt hatten. Reanimationsbedürftige Neugeborene, Neugeborene mit einer Fehlbildung sowie Neugeborene von Risikoschwangeren (z. B. Präeklampsie, prolongierter Blasensprung, Gestationsdiabetes, Raucherinnen, Substanzabusus) schlossen die Forscherinnen und Forscher von der Analyse aus. 672 Neugeborene $(82,3 \%)$ wurden innerhalb der ersten 10 Lebensminuten unbekleidet in Bauchlage auf die nackte Brust der Mutter gelegt und verbrachten in dieser Position 40 Minuten ohne Unterbrechung. Die übrigen 144 Neugeborenen $(17,6 \%)$ wurden nach der Entbindung zunächst von der Mutter getrennt, unter einer Wärmelampe versorgt und anschließend zur Mutter zurückgebracht. Als primären Studienendpunkt definierten die Wissenschaftlerinnen und Wissenschaftler die Hospitalisierung der Neugeborenen vor der Entlassung von der Wöchnerinnenstation.
Das Ergebnis: Die häufigsten Gründe für eine stationäre Behandlungsbedürftigkeit der Neugeborenen stellten im Studienkollektiv der Ikterus (45\%), die Hypoglykämie/andere metabolische Störungen $(31,4 \%)$, Saug- oder Fütterungsschwierigkeiten (18,3\%) sowie der Infektionsverdacht $(3,8 \%)$ dar. Diejenigen Neugeborenen, die postnatal auf der Brust ihrer Mutter liegen durften, mussten signifikant seltener stationär aufgenommen werden als die Neugeborenen ohne Hautkontakt ( 13,8 vs. $26,4 \%$; Relatives Risiko 0,44; $95 \%$ KI 0,29-0,68). Das Geschlecht der Neugeborenen hatte dagegen keinen wesentlichen Einfluss auf die Hospitalisierungsrate. Die logistische Regressionsanalyse ergab: Der Hautkontakt, das Gestationsalter sowie das Geburtsgewicht stellten signifikante Einflussfaktoren bezüglich der stationären Behandlungsbedürftigkeit dar.

FAZIT

Postnatal stabile Neugeborene mit intermediärem und geringem Risiko müssen innerhalb der ersten Lebenstage signifikant seltener stationär aufgenommen werden, wenn sie unmittelbar nach der Geburt über längere Zeit Hautkontakt mit der Mutter haben dürfen, schlussfolgern die Wissenschaftlerinnen und Wissenschaftler. Warum dies so ist, müssen nun ihrer Ansicht nach weitere Untersuchungen klären. Angesichts der Studienergebnisse halten sie das Bonding für eine effektive Präventionsstrategie.

Dr. Judith Lorenz, Künzell 